\title{
A Comparison of the Predictions of a Finite Element Model and Multiscale Model for a Rough MEMS Electrical Contact
}

\author{
Robert L. Jackson+ \\ Hong Liu* \\ Dimitri Leray* \\ +Department of Mechanical Engineering \\ Auburn University, Auburn, Alabama, USA \\ jackson@auburn.edu \\ *Institut Clément Ader, INSA; \\ CNRS, LAAS \\ Univ de Toulouse, INSA, LAAS; Toulouse, France
}

\begin{abstract}
Rough surface contact is difficult to model effectively due to multiple scales of detail that need to be considered. This work presents the results of a multiscale rough surface contact model in comparison to a finite element based deterministic model for the electrical contact of a MEMS microswitch. The real area of contact and electrical contact resistance are predicted and compared as a function of normal load. The results show good quantitative and qualitative correlation between the two methods. As expected, the contact area increases nominally linearly with load, while the contact resistance decreases with load. It is notable though that the contact pressure is up to $16 \%$ higher than the hardness (2.8 times yield strength), and could be even higher for other surfaces.
\end{abstract}

Keywords: rough surface contact, numerical, contact resistance and conductance

\section{INTRODUCTION}

Electrical contact resistance is an important phenomenon which occurs when current is driven between two contacting rough surfaces. The roughness of the surfaces causes only small peaks or asperities to be in contact. The geometry of a typical surface is complicated and possesses features on many different scales.

A variety of electrical contact resistance models have been developed since rough surface contact models were first considered by Archard in 1955 [1]. Archard modeled rough surface contact by stacking smaller asperities on top of larger asperities. Structurally, the asperity scales are in series, while the individual asperities of each scale are in parallel and the load is distributed evenly among them. Archard's model, however, proved difficult to implement because no technique was presented to calculate the size of the asperities at each scale. The work herein uses the Archard stacked asperity concept, further developed by Ciavarella et al. [2-4], Jackson and Streator [5], and Jackson [6].

Archard's concept of multi-scale contact was first employed for modeling contact resistance by Ciavarella et al. [4] for elastic surfaces described by the Weierstrass-Mandelbrot function. Almeida et al. [7] used a fast-Fourier transform (FFT) based multi-scale contact model which included the effect of scale dependent electrical resistance and yield strength to analyze MEMS switches [7, 8]. Wilson et al. [9] improved the multi-scale methodology by modeling asperities using an elastic-plastic sinusoidal contact model. Simplified closed form versions of the models have also been proposed $[6,10,11]$.

Despite the development of these theoretical models, there is relatively little work that attempts to verify them in comparison to other predictions. The focus of the current work is to compare the predictions of the full multiscale electrical contact resistance model in comparison to the results of a deterministic finite element model (FEM). The deterministic model makes no simplifications of the surface profile obtained from measurements. The comparison is made for the case of a rough MEMS electrical contact.

\section{CONTACT MECHANICS}

\section{A. Multi-Scale Perfectly Elastic Contact}

The multi-scale model derived by Jackson and Streator [5] uses a similar approach to Archard [6] but provides a methodology that can be applied to real surfaces with relative ease. A 
fast Fourier transform is first performed on the surface profile data, resulting in a summation of sine and cosine waves. The complex forms of the sine and cosine terms at each frequency are combined using a complex conjugate to provide the amplitude of the waveform at each scale, used for further calculations. Each frequency is considered a scale or layer of asperities which are stacked iteratively upon each other. In equation form this is:

$$
\begin{aligned}
& A=\left(\prod_{i=1}^{i_{\max }} \overline{A_{i}} \eta_{i}\right) A_{n} \\
& P=\overline{P_{i}} \eta_{i} A_{i-1}
\end{aligned}
$$

where $A$ is the real area of contact, $\eta$ is the areal asperity density, $P$ is the contact force, $A_{n}$ is the nominal contact area, and the subscript $i$ denotes a frequency scale, with $i_{\max }$ denoting the highest frequency scale considered. Each frequency scale is modeled using a sinusoidal contact model. Equations derived in [5] and fit to the data given by Johnson, Greenwood, and Higginson [7] are used for this:

$$
\begin{aligned}
& \left(A_{J G H}\right)_{1}=\frac{2 \pi}{f^{2}}\left[\frac{3}{8 \pi} \frac{\bar{p}}{p^{*}}\right]^{2 / 3} \\
& \left(A_{J G H}\right)_{2}=\frac{1}{f^{2}}\left(1-\frac{3}{2 \pi}\left[1-\frac{\bar{p}}{p^{*}}\right]\right)
\end{aligned}
$$

Then for $\frac{\bar{p}}{p^{*}}<0.8$

$$
A=\left(A_{J G H}\right)_{1}\left(1-\left[\frac{\bar{p}}{p^{*}}\right]^{1.51}\right)+\left(A_{J G H}\right)_{2}\left(\frac{\bar{p}}{p^{*}}\right)^{1.04}
$$

and for $\frac{\bar{p}}{p^{*}} \geq 0.8$

$$
A=\left(A_{J G H}\right)_{2}
$$

where $p^{*}$ is the average pressure for complete contact between the surfaces. It is given by [7] as:

$$
p^{*}=\sqrt{2} \pi E^{\prime} \Delta f
$$

\section{B. Multi-Scale Elastic-Plastic Contact}

Many of the asperities at the different frequency scales undergo plastic deformation. An elastic-plastic sinusoidal contact model is needed to consider this effect. The equations used here to calculate the elastic-plastic contact are derived from FEM results by Krithivasan and Jackson [8]. The methodology is similar to the perfectly elastic case but uses a different set of formulas once a calculated critical pressure and area are reached. The critical average pressure and area are given by:

$$
\begin{aligned}
& \bar{p}_{c}=\frac{1}{6 \pi}\left(\frac{1}{B E^{\prime}}\right)^{2}\left(\frac{C \cdot S_{y}}{2}\right)^{3} \\
& A_{c}=\frac{2}{\pi}\left(\frac{C S_{y}}{8 \Delta f^{2} E^{\prime}}\right)^{2}
\end{aligned}
$$

where $C=1.295 \cdot \exp (0.736 v)$.

At low loads, $\bar{p}<\bar{p}_{c}$, and consequently small areas of contact, it is acceptable to assume that the deformation of the contacting asperities will behave perfectly elastically. However, as the load increases past the critical value, plastic deformation will begin to occur within the asperities. To evaluate the plastic deformation we replace Eq. (3) with:

$$
\begin{aligned}
& A_{P}=2\left(\frac{A_{c}}{2}\right)^{\frac{1}{1+d}}\left(\frac{3 \cdot \bar{p}}{4 C S_{y}} \lambda^{2}\right)^{\frac{d}{1+d}} \\
& d=3.8 \cdot\left(\frac{E^{\prime}}{S_{y}} \cdot \frac{\Delta}{\lambda}\right)^{0.11}
\end{aligned}
$$

This replacement results in the following equation for contact area:

$$
A=\left(A_{P}\right)\left(1-\left[\frac{\bar{p}}{p_{P}^{*}}\right]^{1.51}\right)+\left(A_{J G H}\right)_{2}\left(\frac{\bar{p}}{p_{P}^{*}}\right)^{1.04}
$$

Where $p_{p}^{*}$ is the average pressure required to obtain complete contact between sinusoidal surfaces when plasticity occurs and is given by [12]:

$$
\begin{aligned}
& \frac{p_{P}^{*}}{p^{*}}=\left(\frac{11}{4 \cdot \Delta / \Delta c+7}\right)^{3 / 5} \\
& \Delta c=\frac{\sqrt{2} \cdot S_{y} \exp \left(\frac{2 v}{3}\right)}{3 \pi E^{\prime} f}
\end{aligned}
$$

For the multiscale model, a description of the geometry at different scales is required. This is obtained by taking an FFT of the surface. In past works, usually only a single slice or line of the profile was considered. Since an entire 3-D surface is used here, the following procedure is followed. The FFT of each column ( $x$ direction) and row ( $y$ direction) is calculated. The complex conjugate of each scale term is used to calculate a single amplitude and to remove the phase term 
(this is identical to previous works). Then the amplitudes for each scale in the $x$ and $y$ directions are averaged to provide a single effective amplitude at each scale. The effective amplitude of each scale is then normalized by the wavelength to give the $B$ ratio at each scale.

In the current work the geometry of a contacting microswitch from a MEMS device as measured by an Atomic Force Microscope (AFM) is considered (see Fig. 1). The area is $2.1 \mu \mathrm{m} \mathrm{x}$ $2.1 \mu \mathrm{m}$. As described, the surface is transformed into the frequency domain using a FFT. These resulting spectrums in the $x$ and $y$ directions and then averaged are shown in Fig. 2. The averaged spectrum is used in the multiscale contact model in the current work.

\section{CONTACT RESISTANCE}

Our focus here is to predict the electrical contact resistance between surfaces having multiple scales of roughness. Since only a few scattered asperities are actually in contact for any given load, the current is restricted to small contact patches. As the current flows through the asperity peaks, it will be effectively "bottlenecked" and result in electrical resistance.

Holm [10] provides a simple formula to calculate the electrical resistance due to asperity contact.

$$
R_{i}=\frac{\rho}{2 a_{i}}
$$

where $R$ refers to the contact resistance, $a$ is the radius of contact, $\rho$ is the electrical resistivity of the surface, and $i$ denotes the scale. This equation is valid only for an asperity in the continuum/ macro-scale or well above the atomic scale. It is known that as the scale decreases, that the mechanical, electrical and thermal properties of the asperity material can change from the bulk properties at large scales. Although as some recent work has shown, these scale dependent effects may not always be influential on electrical and thermal contact resistance [7, 8, 10, 13-15].

As discussed in section 2, the multi-scale model is an iterative method that calculates area and resistance for each particular scale. The first step is to calculate the average radius of contact at each scale $i$ :

$$
a_{i}=\sqrt{\frac{A_{i}}{2 \cdot \pi \cdot A_{i-1} \cdot f^{2}}}
$$

Once the contact radius is established, Eq. (16) is used to calculate the resistance of each asperity at each scale. Oftentimes, an alleviation factor is used in thermal contact resistance calculations to account for the effect of a large

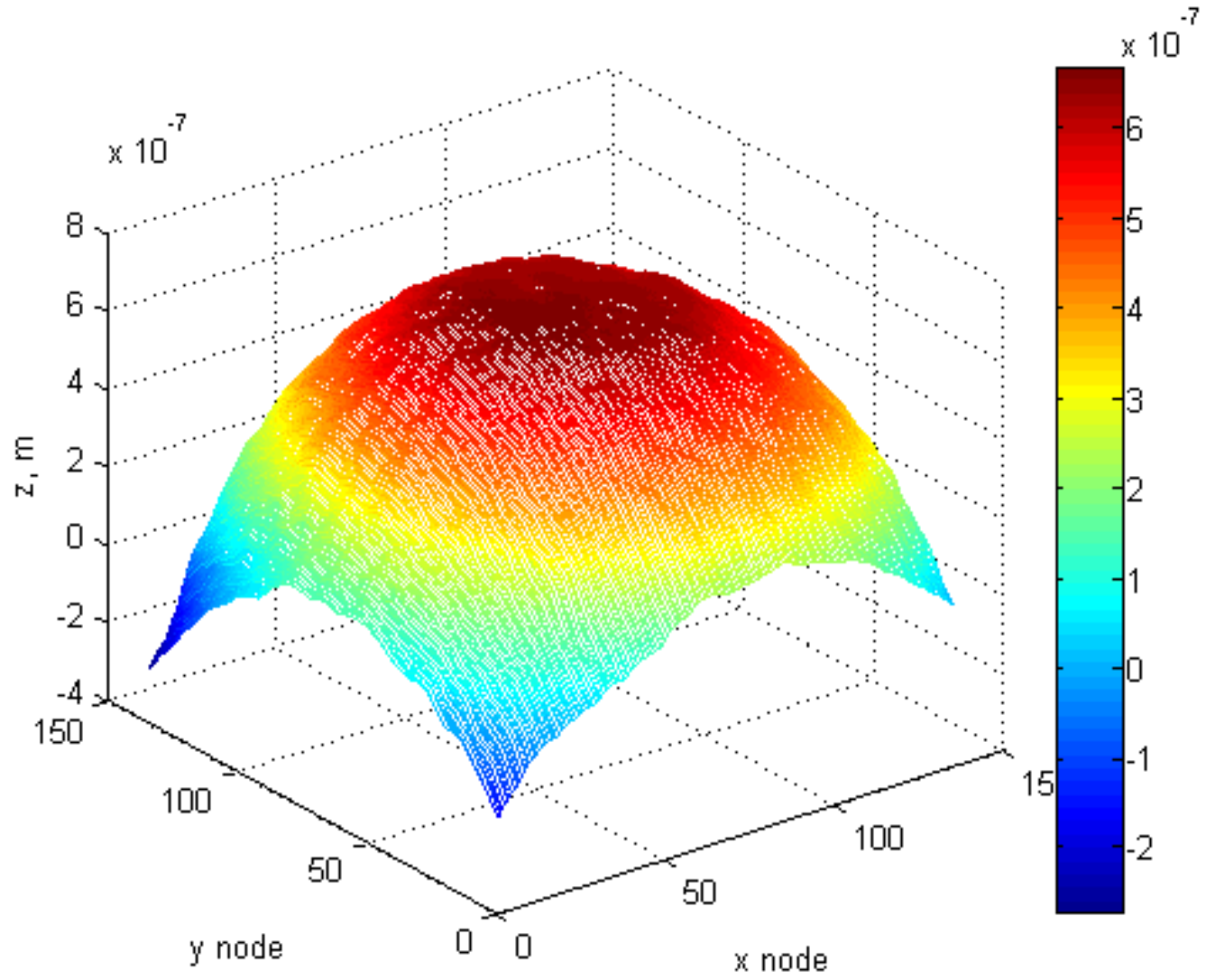

Figure 1: AFM profile of the MEMS microswitch. 


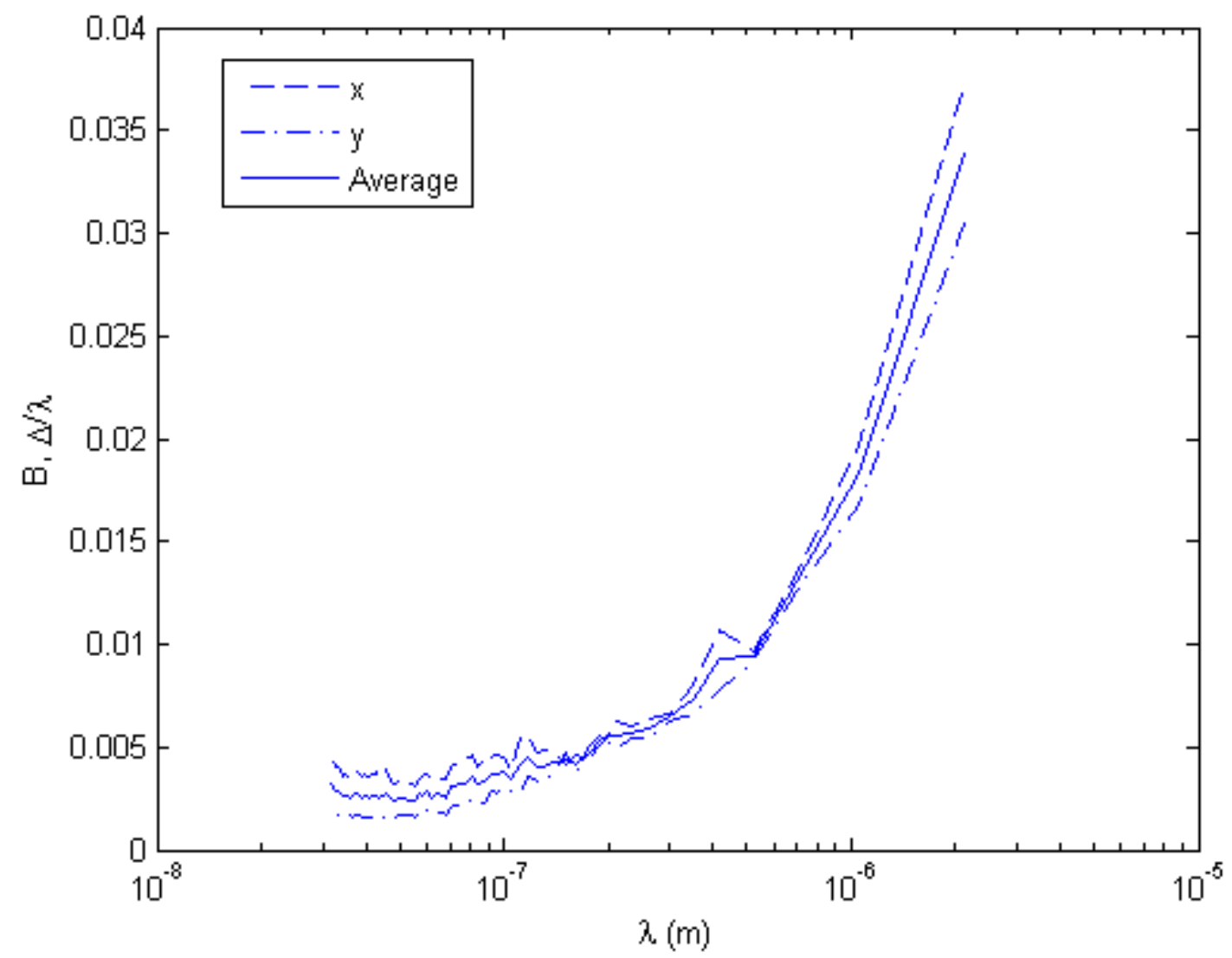

Figure 2: Spectrum of the MEMS surface by averaging the terms at each scale obtained from an FFT of each row (x) and column (y) of data, and then taking an overall average.

contact area in relation to the apparent area. Since electrical and thermal contact resistances are similar, it is reasonable to presume that the alleviation factor, $\Psi$, should also be included for electrical contact resistance. The simplified version of the factor offered by Cooper et al. [12] is used in this work:

$$
\Psi_{i}=\left(1-\sqrt{A_{i} / A_{i-1}}\right)^{1.5}
$$

Therefore, the total resistance at each scale is found by summing the reciprocal of the resistance of each individual asperity on each scale. Then the alleviation factor is multiplied by this resistance at each scale. Finally, this value is then summed over all the considered scales to find the total resistance for the entire surface in contact:

$$
R_{t o t}=\sum_{i=1}^{i_{\max }}\left(\frac{R_{i}}{n_{i}} \psi_{i}\right)
$$

Note that $n_{i}$ is the number of asperities in contact at each scale, $i$. Since two peaks are present in a square wavelength of area, $n$ is given by

$$
n_{i}=2 \frac{A_{i-1}}{\lambda_{i}^{2}}
$$

However, it is not straight forward to calculate the total number of asperities in contact using the multiscale model. This is because at the smallest asperity scales that are under very high pressures, they will be nearly flattened out with only a few pockets of non-contacting areas. Therefore it is difficult to discern overall what are actually separate asperity contacts. In the current work we derive an approximate prediction by considering the contact geometry of sinusoidal surfaces. In sinusoidal contact, the contact area only splits into separate asperities when the contact area is less than half of the nominal area. Assuming that this geometrical trend occurs over all the scales of the rough surface in contact, the following relation is found:

$$
(1 / 2)^{n}=A / A_{n}
$$

Where $\mathrm{n}$ is the number of asperities, which solving for $n$ results in

$$
n=\frac{\ln \left(A / A_{n}\right)}{\ln (0.5)}
$$

\begin{tabular}{|c|c|}
\hline $\begin{array}{l}\text { Table 1: } \\
\text { Material properties of gold used in this work }\end{array}$ \\
\hline$E=80 \cdot 10^{9} \mathrm{~Pa}$ & $S_{y}=300 \cdot 10^{6} \mathrm{~Pa}$ \\
\hline$v=0.42$ & $\rho=2.21 \cdot 10^{-8} \Omega \mathrm{m}$ \\
\hline$E_{t}=10 \cdot 10^{9} \mathrm{~Pa}$ & \\
\hline
\end{tabular}

\section{FINITE ELEMENT MODEL}

To compare with the multiscale methodology described in the previous section, the present study uses a deterministic finite element method to also solve the problem. Atomic force microscopy (AFM) is used to measure the topography of a microswitch bump, which results 


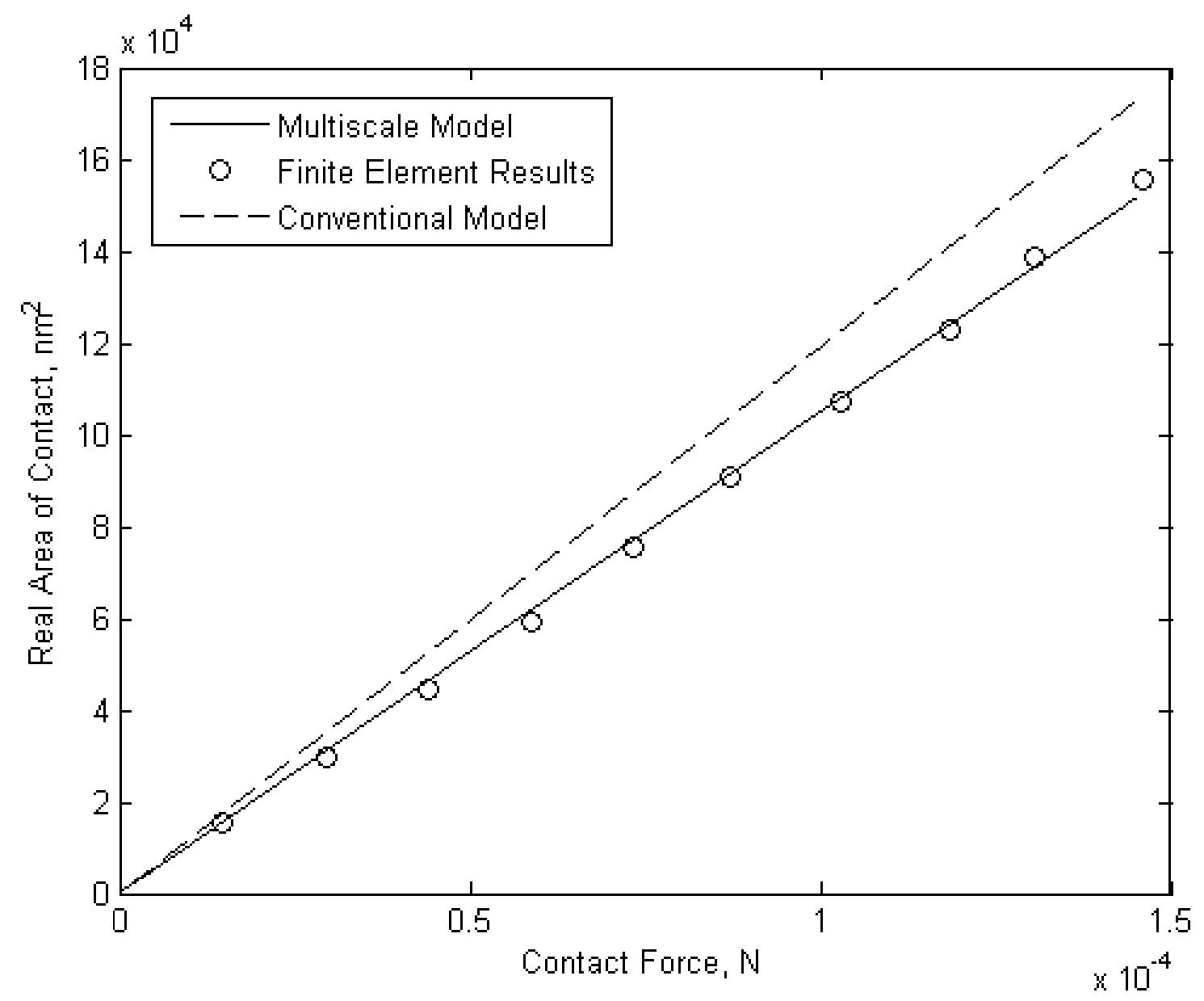

Figure 3: Comparison of the real area of contact predictions made by the finite element model, the multiscale model and a conventional model.

in a precision profile of the surface, as shown previously in Fig. 1. Using the AFM scan data, the finite element (FEM) contact model is created in the commercial ANSYS package 11.0. The area considered by the FEM is reduced to $1.2 \mu \mathrm{m} \mathrm{x}$ $1.2 \mu \mathrm{m}$ to improve computational efficiency. Nonetheless, the model employs over 500,000 elements, uses contact elements on the surface, and assumes that the base is held stationary. For more details of the finite element model, please see the previous work[16].

The material properties listed for gold in Table 1 are used for all of the calculated results presented in this work.

\section{RESULTS}

The load is incrementally increased in the FEM model until it reaches $145 \mu \mathrm{N}$. The multiscale model is also used to make predictions over the same range of loads. The predictions of the real contact area for both are shown in Fig. 3. The results from both models show the same nearly linear change in the real contact area as a function of load, and also quantitatively compare very well. Note that this confirms the nearly linear trend, of many previous rough surface contact models as well $[1,17,18]$. Also shown is a conventional model that calculates the contact pressure as the hardness $\left(2.8 \cdot S_{y}\right)[19,20]$. The conventional calculation tends to over predict the contact area in comparison to the multi-scale model and finite element model.

Since the predictions of the real contact area versus load are very similar, it is expected that the average real contact pressures for the two methods will also be similar. This is calculated by dividing the contact force by the real contact area. Once this is achieved, it is evident that the real contact pressures for the two models are very similar (see Fig. 4). It is also worth noting that the pressure is actually greater than the value of $2.8 \cdot S_{y}$ that is often rounded up to $3 \cdot S_{y}$ and used to approximate the real contact area[21]. The contact pressure is up to $16 \%$ higher than $2.8 \cdot S_{y}$, As is noted in other works, for some surfaces this pressure can even be much greater than $3 \cdot S_{y}[6,12,22-28]$ or sometimes even less [29-31]. Most rough surface contact models do not succeed at predicting this.

Next the predictions of the electrical contact resistance between the multiscale model and the finite element results are compared (see Fig. 5). Here, two versions of the models are considered. The first neglects any scale effect on resistance 


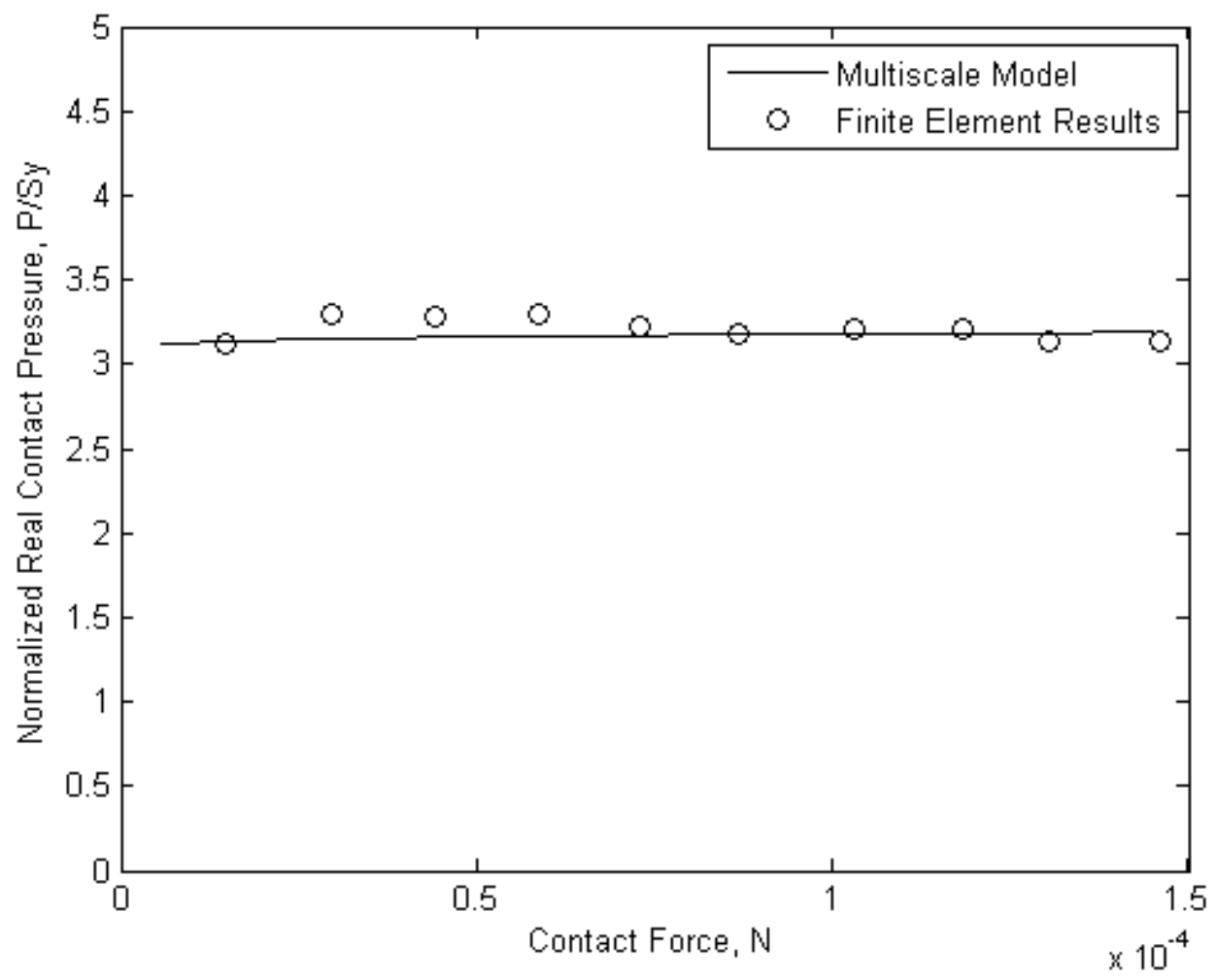

Figure 4: Compatison of the real contact force predictions made by the firite element model and multiscale model.

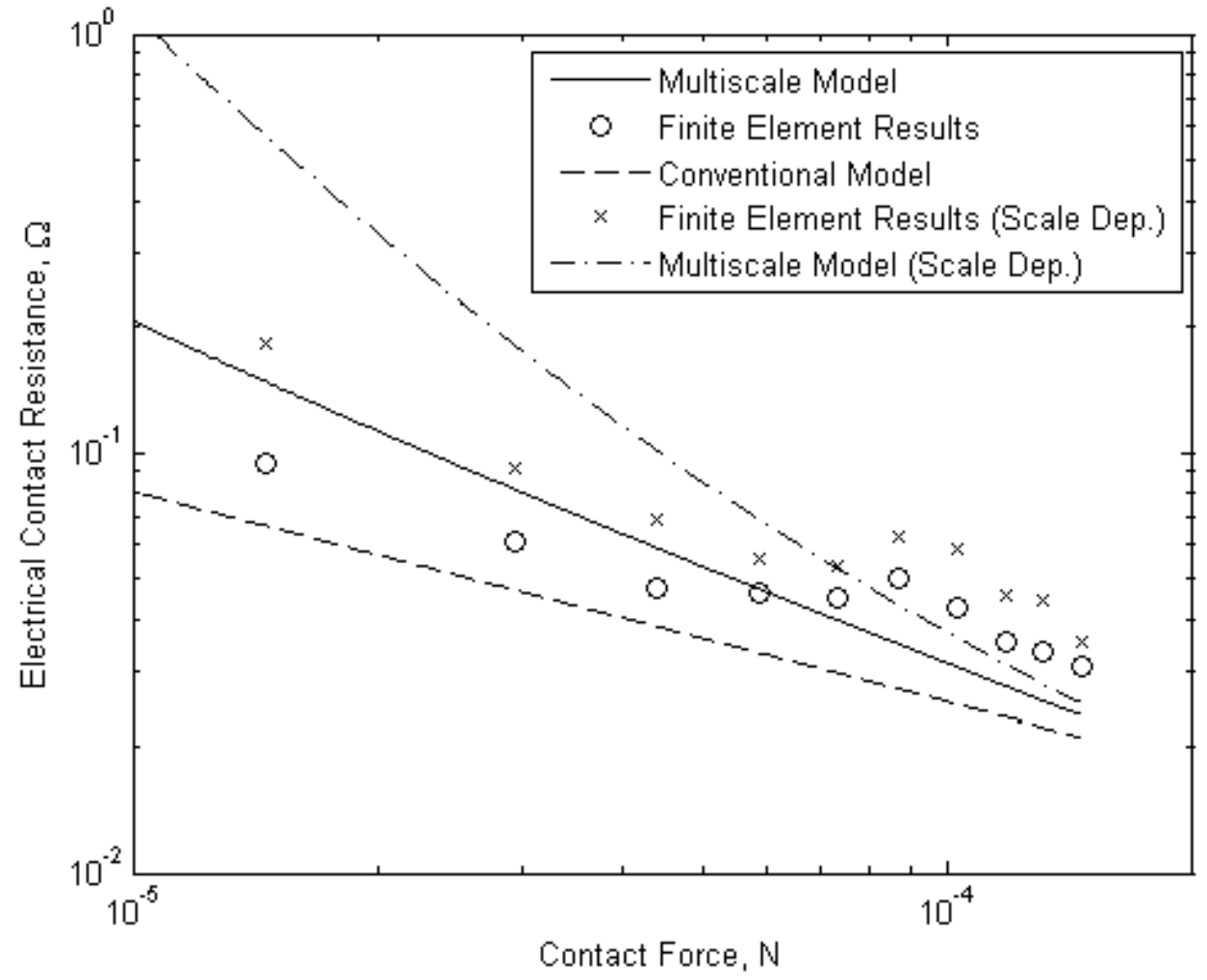

Figure 5: Comparison of the electrical contact resistance predictions made by the finite element model and multiscale model. 


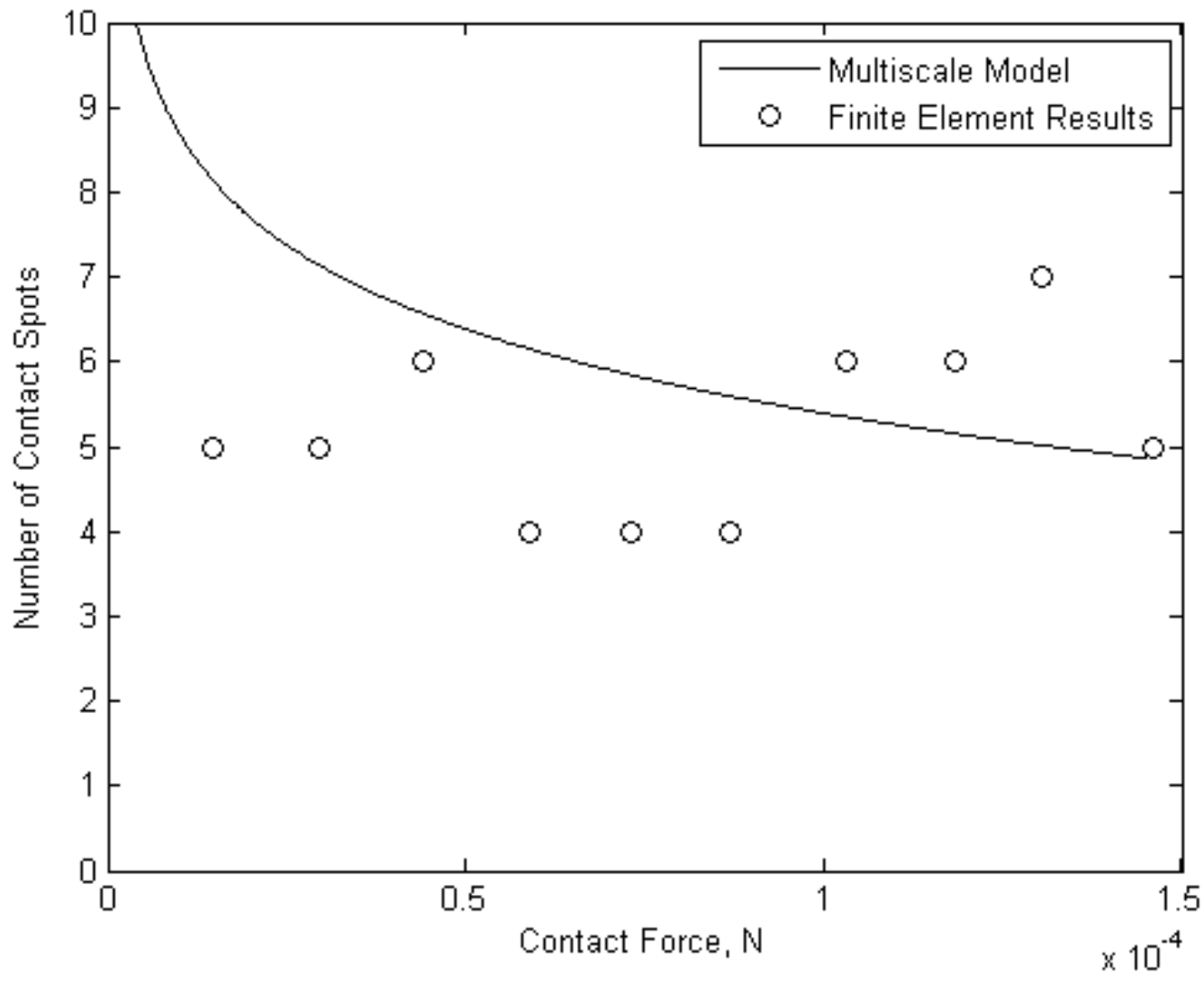

Figure 6: Comparison of the number of contact spots as predicted by the finite element model and multiscale model.

(i.e. Holm resistance as described previously in this work) and the second considers the transition of electrical conduction from a diffusive mechanism to a ballistic one (i.e. quantum). The method of Wexler[32] as described in Timsit[15] and Jackson et al. [14] are used to consider this (these results are labeled as 'Scale Dep.' in Fig. 5). For brevity this methodology is not described in detail here. However, it should be noted that in the current work the scale effect in the multiscale model is considered on the final results using Eqs. (19 and 22), while in [14], the scale effect is included at each scale of roughness iterively. For contact resistance, the quantitative comparison is not as good between the FEM and multiscale model as with the contact area. However, the qualitative trend is still the same, and the average trend of the FEM model seems to follow the multiscale model. It should also be noted, that as expected, the scale dependent resistance effects increase the overall contact resistance as shown in Fig. 5.

To help illustrate the advantages of the finite element method and the multiscale model the results are also compared to a conventional model. The conventional model assumes that the pressure is the hardness $\left(2.8 \cdot S_{y}\right)$ and that the contact spots are all identical and evenly distributed[19, 20]. It is difficult to predict the number of contact spots using the conventional approach, so we use the mean value of 5 predicted by the FEM (Eq. 22 could also be used and Malucci also provides some methods $[11,28,33])$. In equation form the conventional model is:

$$
R=\frac{\rho \sqrt{\pi \cdot 2.8 \cdot S_{y}}}{2 \sqrt{n \cdot F}}
$$

The predictions of the conventional model given by Eq, (23) are shown in Fig. 5 alongside the multiscale model and the FEM results. Note that although Eq. 23 predicts the same trend as the other models, it under-predicts the resistance at all loads. This is because of the assumption that all the contacts are identical in size and that hardness is the average real contact pressure.

Finally, the predictions of the newly derived approximate equation for the number of contact spots (Eq. 22) is compared to the FEM predictions (see Fig. 6). Eq. 22 makes a prediction that is fractional, while the number of spots is actually an integer. Note that Eq. 22 is not used in calculating the contact resistance for the multiscale model and is only meant to provide an overall prediction of the number of contact spots. Although the results are not the same and the trends are different, they do show a similar average value. Note that the counting of contact spots can sometimes be 
subjective, especially when two adjacent spots just begin to coalesce. This is shown by Fig. 7, which is a plot of the contact pressure as predicted by the FEM model.

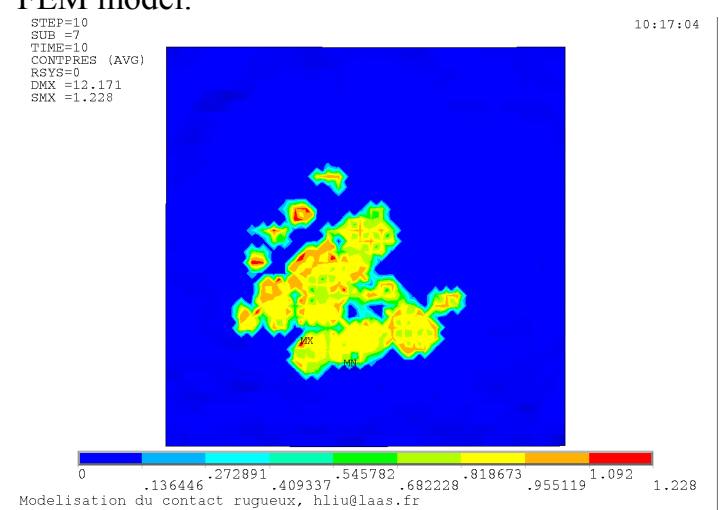

Figure 7: Contact pressure as predicted by FEM.

\section{CONCLUSIONS}

This work presents the results of a multiscale based electrical contact resistance model in comparison to a finite element model of a MEMS microswitch contact. The results compare qualitatively well for all the predicted quantities. The models appear to be almost equivalent in predicting the contact mechanics as the real contact area and real contact pressure predictions are almost identical. It should be noted that both models predicted an average real contact pressure that is higher than $2.8 \cdot S_{y}$. However, the models differed more when making predictions of the electrical contact resistance, even though on average they were very similar. This is probably due to the location, distribution and number of contact spots possibly differing between the models. It should be noted that in real contact situations that contact spots usually do not appear as evenly distributed circular spots. Due to this it can be difficult to quantitatively characterize these values. Nonetheless, the overall contact resistance results of the two models do appear to compare reasonably well. This is also demonstrated by the lower resistance predictions of the conventional model that assumes that all of the contact spots are identical in size. Finally, the effect of scale dependent resistance was also considered and shows to increase all the resistance predictions by a significant amount.

\section{ACKNOWLEDGEMENTS}

Also, thanks to Adrien Broue for providing the

AFM scan data.

\section{REFERENCES}

[1] J. F. Archard, "Elastic Deformation and the Laws of Friction," Proc. R. Soc. Lond. A vol. 243, pp. 190-205, 1957.
[2] M. Ciavarella and G. Demelio, "Elastic Multiscale Contact of Rough Surfaces: Archard's Model Revisited and Comparisons with Modern Fractal Models," J. Appl. Mech., vol. 68, pp. 496-498, 2001.

[3] M. Ciavarella, G. Demelio, J. R. Barber, and Y. H. Jang, "Linear elastic contact of the Weierstrass Profile," Proc. R. Soc. Lond. A, pp. 387-405, 2000.

[4] M. Ciavarella, G. Murolo, and G. Demelio, "The Electrical/Thermal Conductance of Rough Surfaces--the Weierstrass-Archard Multiscale Model," Int. J. Solids and Structures, vol. 41, pp. 4107-4120, 2004.

[5] R. L. Jackson and J. L. Streator, "A Multiscale Model for Contact between Rough Surfaces," Wear, vol. 261, pp. 1337-1347, 2006.

[6] R. L. Jackson, "An Analytical Solution to an Archard-type Fractal Rough Surface Contact Model," Trib. Trans., vol. 53, pp. 543 - 553, 2010.

[7] L. Almeida, R. Ramadoss, R. Jackson, K. Ishikawa, and Q. Yu, "Study of the electrical contact resistance of multi-contact MEMS relays fabricated using the MetalMUMPs process," J. of Micromech. Microeng., vol. 16, pp. 1189-1194, 2006.

[8] L. Almeida, R. Ramadoss, R. Jackson, K. Ishikawa, and Q. Yu, "Laterally actuated multicontact MEMS relay fabricated using MetalMUMPS process: experimental characterization and multiscale contact modeling," J. Micro/Nanolith. MEMS MOEMS, vol. 6, p. 023009., 2007.

[9] W. E. Wilson, S. V. Angadi, and R. L. Jackson, "Surface Separation and Contact Resistance Considering Sinusoidal ElasticPlastic Multi-Scale Rough Surface Contact," Wear, vol. 268, pp. 190-201, 2010.

[10] R. L. Jackson, H. Ghaednia, Y. A. Elkady, S. H. Bhavnani, and R. W. Knight, "A ClosedForm Multiscale Thermal Contact Resistance Model," IEEE Trans. Comp. Packag. Technol., vol. 2, pp. 1158-1171, 2012.

[11]R. L. Jackson, R. D. Malucci, S. Angadi, and J. R. Polchow, "A Simplified Model of Multiscale Electrical Contact Resistance and Comparison to Existing Closed Form Models," in Electrical Contacts, 2009 Proceedings of the 55th IEEE Holm Conference on, 2009, pp. 28-35.

[12]R. L. Jackson, V. Krithivasan, and W. E. Wilson, "The Pressure to Cause Complete Contact between Elastic Plastic Sinusoidal Surfaces," IMechE J. of Eng. Trib. - Part J., vol. 222, pp. 857-864, 2008.

[13]R. L. Jackson, S. H. Bhavnani, and T. P. Ferguson, "A Multi-scale Model of Thermal Contact Resistance between Rough Surfaces," 
ASME J. Heat Transfer, vol. 130, p. 081301, 2008.

[14]R. L. Jackson, E. R. Crandall, and M. J. Bozack, "An Analysis of Scale Dependent and Quantum Effects on Electrical Contact Resistance between Rough Surfaces," in Electrical Contacts (Holm), 2012 IEEE 58th Holm Conference on, 2012, pp. 1-10.

[15]R. S. Timsit, "Electrical Conduction Through Small Contact Spots," presented at the The 50th IEEE Holm Conference on Electrical Contacts, Seattle, WA, USA, 2004.

[16] H. Liu, D. Leray, S. Colin, P. Pons, and A. Broue, "Finite Element Based Surface Roughness Study for Ohmic Contact of Microswitches," in Electrical Contacts (Holm), 2012 IEEE 58th Holm Conference on, 2012, pp. 1-10.

[17] J. A. Greenwood and J. B. P. Williamson, "Contact of Nominally Flat Surfaces," Proc. R. Soc. Lond. A, vol. 295, pp. 300-319., 1966.

[18]R. L. Jackson and I. Green, "A Statistical Model of Elasto-plastic Asperity Contact between Rough Surfaces " Trib. Int., vol. 39, pp. 906-914, 2006.

[19] J. A. Greenwood, "Constriction Resistance and the Real Area of Contact," Brit. J. Appl. Phys., vol. 17, pp. 1621-1632, 1966.

[20]R. Holm, Electric Contacts. New York: Springer, 1967.

[21]D. Tabor, The Hardness of Materials. Oxford: Clarendon Press, 1951.

[22]Y. F. Gao, A. F. Bower, K. S. Kim, L. Lev, and Y. T. Cheng, "The behavior of an elasticperfectly plastic sinusoidal surface under contact loading," Wear, vol. 261, pp. 145-154, 2006.

[23] Y. F. Gao and A. F. Bower, "Elastic-plastic contact of a rough surface with Weierstrass profile," Proc. R. Soc. A, vol. 462, pp. 319348, 2006.
[24]W. Manners, "Plastic Deformation of a Sinusoidal Surface," Wear, vol. 264, pp. 6068, 2008.

[25] V. Krithivasan and R. L. Jackson, "An Analysis of Three-Dimensional Elasto-Plastic Sinusoidal Contact," Trib. Letters, vol. 27, pp. 31-43, 2007.

[26] J. B. P. Williamson and R. T. Hunt, "Asperity Persistance and the Real Area of Contact Between Rough Surfaces," Prod. Roy. Soc. (London) A, vol. 327, pp. 147-157, 1972.

[27] J. Pullen and J. B. P. Williamson, "On the Plastic Contact of Rough Surfaces," Proc. R. Soc. Lond. A, vol. 327, pp. 159-173, 1972.

[28]R. D. Malucci and F. R. Ruffino, "Materials Considerations in Using Voltage Drop for Power Rating," in IEEE Holm Conference on Electrical Contacts, Pittsburgh, PA, 2007, pp. 25-31.

[29] L. Kogut, and Komvopoulos, K., "Analysis of Spherical Indentation Cycle of ElasticPerfectly Plastic Solids," J. Mater. Res., vol. 19, pp. 3641-3653, 2004.

[30] J. J. Quicksall, R. L. Jackson, and I. Green, "Elasto-plastic hemispherical contact models for various mechanical properties," IMechE $J$. of Eng. Trib. - Part J., vol. 218, pp. 313-322, 2004.

[31] S. S. Wadwalkar, R. L. Jackson, and L. Kogut, "A study of the elastic-plastic deformation of heavily deformed spherical contacts " IMechE Part J, J. of Eng. Tribology, vol. 224, pp. 1091-1102, 2010.

[32] G. Wexler, "The size effect and the non-local Boltzmann transport equation in orifice and disk geometry," Proc. Phys. Soc., vol. 89, pp. 927-941, 1966.

[33] R. D. Malucci, "Multi-spot model showing the effects of nano-spot sizes," in IEEE Holm Conference on Electrical Contacts, 2005, pp. 291-297. 